\title{
Comparison of heath communities from the class Nardo-Callunetea from the Torun Basin and other regions of Poland
}

\author{
*Joanna Chojnacka, *Wiesław Cyzman, **Andrzej Nienartowicz, **Miłosz Deptuła \\ * Department of Taxonomy and Plant Geography, Institute of Ecology and Environment Protection, \\ ** Laboratory of Ecological Processes Modelling, Institute of Ecology and Environment Protection, \\ Faculty of Biology and Earth Sciences, Nicolaus Copernicus University, \\ Gagarina 9, 87-100 Toruń, Poland \\ e-mail: anienart@umk.pl
}

\begin{abstract}
This paper presents the variability within two heath associations, i.e. Pohlio-Callunetum and Arctostaphylo-Callunetum in the territory of Poland. The variability of the former syntaxon was determined based on 9 sets of published relevés, made in different regions of Poland. In the case of the latter syntaxon, there were 13 sets of relevés. The sets were being compared in respect of species composition assuming the degrees of species constancy as characteristics of the compared sets. The comparisons have been done with the classic phytosociological method according to Braun-Blanquet, as well as with numerical taxonomy methods using the software MVSP. In both cases, the subsets of relevés from the artillery range near the city of Torun were clearly separated from the others. In the obtained dendrograms, relevés from the neighbourhood of Torun, classified within the analysed subassociations formed separate clusters and their distance to other groups of relevés from the same association but from different regions of Poland was significant. Both in the case of the association Pohlio Callunetum and the association Arctostaphylo-Callunetum, sets of relevés from the Skwierzyna (coniferous) Forest in western Poland and from the Tuchola Forest in northern Poland were the most similar to relevés from the neighbourhood of Torun.
\end{abstract}

Key words: characteristic species combination, constancy of species, numerical classification, plant association, sets of phytosociological records, syntaxonomy.

\section{Introduction}

The range of dry heaths from the class Nardo-Callunetea comprises almost the whole territory of Poland. According to maps included by Kujawa-Pawlaczyk (2003) in the „Guidebook to Natura 2000 areas”, mountainous areas in southern Poland are the only place they do not occur. The two main associations of dry heaths, i.e. Pohlio-Callunetum and Arctostaphylo-Callunetum cover a considerable part of our country, except for the mountains, but their main occurrence centres are located in different parts of Poland. The occurrence centre of the association Pohlio-
Callunetum is located in the western part of our country. Whereas eastern Poland is the occurrence centre of the association Arctostaphylo-Callunetum. According to Matuszkiewicz (2007), the occurrence area of the more continental association of bearberry heath is situated outside the Polish borders, in Belarus.

The variability of the association of nodding threadmoss heaths Pohlio-Callunetum is poorly researched in Poland. Communities of this syntaxon from the Skwierzyna Forest were described by Balcerkiewicz and Brzeg (1989). They regard the associations classified within the alliance of Pohlio-Callunion as substitute communities for pine forests, particularly for Leucobryo-Pinetum. 
According to Balcerkiewicz and Brzeg (1989), PohlioCallunetum is the most common community from the order Calluno-Ulicetalia in the Skwierzyna Forest. According to both authors, it is a separate plant association despite the species from the class Vaccinio-Piceetea, which encroach on its patches and their high similarity to the herb layer of pine forests.

Also the association of Arctostaphylo-Callunetum so far has been hardly investigated in Poland. Z dotychczas publikowanych zdjęć fitosocjologicznych tego syntaksonu niewiele zostało wykonanych na poligonach wojskowych na obszarze Polski. A note on bearberry patches surrounded by patches of Corynephorus canescens and Calluna vulgaris on dunes near Torun was provided by Werdyn (1964). However, no relevés from these areas were included in the aforementioned publication. The research on the structure of bearberry heaths by the method of relevés on the artillery range near the city of Torun, was conducted by W. Gugnacka-Fiedor and E. Adamska in 1998-2001, as well as by K. Chojnacka in 2008. The results of this research are presented in the separate articles included in the 12th Issue of Ecological Questions.

Until recently, there were not too many descriptions of the syntaxon Arctostaphylo-Callunetum from the areas other than military training grounds in Poland. Only the research by Ciosek (2000) in central eastern Poland considerably increased the collection of relevés from this association. It is a well-known fact, however, that in Poland bearberry occurs not only in open communities but also in forest communities, mainly in pine forests. Fijałkowski and Górski (1968) recorded the presence of Arctostaphylos uva-ursi in the area of Polesie, the south-eastern part of Poland. Bearberry grew here in the mixed coniferous forest of Pino-Quercetum festucetosum ovinae and in the pine forest of Pino-Vaccinietum myrtilli. Furthermore, in this region the species was also observed in psammophilous grasslands classified as the associations of Corynephoretum canescentis and Festuco-Koelerietum. Whereas, the aforementioned authors did not report the occurrence of bearberry heaths Arctostaphylo-Callunetum in the study area.

Also Gawłowska (1964) in her study on the distribution, resources and protection of kinnikinnick bearberry in Poland quoted this species only as a species characteristic of natural pine forests Pineto-Vaccinietum myrtilli. After Braun-Blanquet et al. (1939), she distinguished e.g. the subassociation Pineto-Vaccinietum myrtilli arctostaphyletosum.

Both types of the discussed heath associations occur in the vicinity of Torun (Chojnacka et al. 2009). According to the map by Kujawa-Pawlaczyk (2003), the city of Torun is situated on the eastern outskirts of the area defined as the occurrence centre of Pohlio-Callunetum and the western outskirts of the occurrence centre of Arctostaphylo-Callunetum. Probably, the extensive range of occurrence of both syntaxa is characterized by the variability of species composition, abundance and characteristic species combinations within both units.

The present paper aims at defining the range of this variability by comparing the sets of relevés from different regions of Poland and made by different authors, classified within both discussed associations. The comparisons were done with both the classic phytosociological method of Braun-Blanquet and methods of numerical taxonomy.

\section{Materials and methods}

For the association of Pohlio-Callunetum, the following sets of relevés were included in the performed classic and numerical phytosociological analyses:

1. Pohlio-Callunetum, the variant with Dianthus carthusianorum, the subvariant with Molinia caerulea - 10 relevés (Chojnacka 2009; Chojnacka et al. 2010) from the artillery range near Torun in Torun Basin [the code applied in dendrograms: PC-v.Dc-s.Mc-TB],

2. Pohlio-Callunetum, the variant with Dianthus carthusianorum, the typical subvariant, the form with Pleurozium schreberi - 12 relevés (Chojnacka 2009; Chojnacka et al. 2010) from the artillery range near Torun [the code: PC-v.Dc-s.t-f.Ps-TB],

3. Pohlio-Callunetum, the variant with Dianthus carthusianorum, the typical subvariant - 21 relevés (Chojnacka 2009; Chojnacka et al. 2010) from the artillery range near Torun [the code: PC-v.Dc-s.t-TB],

4. Pohlio-Callunetum, the typical variant, the typical form - 23 relevés (Chojnacka 2009; Chojnacka et al. 2010) from the artillery range near Torun [the code: PC-v.t-f.t-TB],

5. Pohlio-Callunetum, the typical variant, the form with Pleurozium schreberi - 7 relevés (Chojnacka 2009; Chojnacka et al. 2010) from the artillery range near Torun [the code: PC-v.t-f.Ps-TB],

6. the Skwierzyna Forest - 13 relevés (Balcerkiewicz \& Brzeg 1993) [the code: PC-SF],

7. the community with Calluna vulgaris, the Tuchola Forest -8 relevés (Meller 1994) made near the villages of Lubnia and Wiele [the code: c.Cv-TF],

8. the community with Calluna vulgaris, the Upland of Dobrzyń - 5 relevés of Kępczyński (1965) [the code: c.Cv-UD],

9. Pineto-Callunetum, the Kampinos Forest - 18 relevés of Kobendza (1930) [the code: PinC-KF].

In the numerical analysis of the association Arctostaphylo-Callunetum, 3 sets of relevés from the artillery range near Torun were included and 10 sets of relevés from other regions of Poland, made in the studied habitat as well as other habitats. These were the following sets of relevés: 
1. Arctostaphylo-Callunetum, the typical variant 5 relevés (Chojnacka 2009; Chojnacka et al. 2010) from the artillery range near Torun [the code: AC-v.t- TB],

2. Arctostaphylo-Callunetum, the variant with Thymus serpyllum - 6 relevés (Chojnacka 2009; Chojnacka et al. 2010) from the artillery range near Torun [the code: AC-v.Ts-TB],

3. Arctostaphylo-Callunetum - 20 relevés (GugnackaFiedor \& Adamska 2010) from the artillery range near Torun [the code: AC-TB]

4. Arctostaphylo-Callunetum cladonietosum - 25 relevés (Ciosek 2000) from the Central-Eastern Poland [the code: AC-cla-CE],

5. Arctostaphylo-Callunetum typicum 29 relevés (Ciosek 2000) from the Central-Eastern Poland [the code: ACtyp-CE],

6. Arctostaphylo-Callunetum thymetosum serpylli 33 relevés (Ciosek 2000) from the Central-Eastern Poland [the code: AC-thy-CE],

7. Arctostaphylo-Callunetum vaccinietosum myrtilli 36 relevés (Ciosek 2000) from the Central-Eastern Poland [the code: AC-vac-CE],

8. Białystok, Ełk- 8 relevés (Faliński \& Bartel 1965) [the code: AC-NE],

9. Central and North-Eastern Poland, and Russia 7 relevés of Tüxen, Ellenberg and Hölscher received from the work of Faliński (1965) [the code: ACP\&R],

10. Green Kurpie Forest -6 relevés (Faliński 1965) [the code: AC-GKF],

11. the Lublin Region, S-E Poland - 10 relevés (Fijałkowski 1993) [the code: AC-LR],

12. Skwierzyna Forest -5 relevés (Balcerkiewicz \& Brzeg 1993) [the code: AC-SF],

13. Tuchola Forest -8 relevés Meller (1994) made near the villages of Lubnia and Wiele [the code: AC-TF].

Due to a small number of relevés, the following tables were not included in numerical analyses: 4 relevés from Augustów and Białowieża Forests by Faliński (1965); 3 relevés from Opole Silesia by Spałek (1996), as well as 2 relevés from the Zadroże Dune near Toruń by Anikiejówna and Gorska (1949). These relevés were included in comparisons carried out with the classic Braun-Blanquet method.

The sets of relevés included in the numerical analysis were compiled in two phytosociological tables. For better clarity, only species with a high frequency were included in the tables, as well as protected and rare species. Species with a very low frequency and species occurring only in 1-2 sets of relevés were omitted. In the table of the association Pohlio-Callunetum, for our relevés from the artillery range near Toruń, the species constancy was presented at the level of variants and at the level of lower syntaxonomic units, i.e. subvariants or forms of the association.
Numerical classification was performed with the software MVSP (Kovach 1985-1999). The compared objects were tables of relevés made in the aforementioned regions. When describing the objects in dendrograms, the abovementioned codes of areas and syntaxa were applied. Constancy degrees were used as characteristics of the compared objects. By applying the twenty-percent ranges denoted with numbers I - V and by taking into account no occurrence of a taxon in a table, they took on values from 0 to 5. No data transformation was done. All taxa were included in the calculations as characteristics of the compared objects. There were 171 characteristics in the case of the association Pohlio-Callunetum, and 198 characteristics in the case of the association Arctostaphylo-Callunetum. Minimum variance methods, the median method and UPGMA were applied as a grouping method (Kovach 1985-1999; Piernik 2008). In the first case, the Squared Euclidean Distance was used as a measure of distance between objects (phytosociological tables), in the second case - Gower's General Similarity Coefficient, and in the third one - the Percent Similarity.

\section{Results}

\subsection{Species composition and characteristic species combination in subsets of relevés}

The comparison of objects classified with the classic Braun-Blanquet method as Pohlio-Callunetum is provided in Table 1, in which the constancy degree of 100 taxons in 11 subsets of relevés is presented. This association is characterized by the domination of common heather Calluna vulgaris, as well as numerous and frequently abundant contribution of species from the alliance Pohlio-Callunion, such as: nodding thread-moss Pohlia nutans, dicranum moss Dicranum scoparium, cup lichens - Cladonia deformis and Cladonia chlorophaea and others. Apart from Calluna vulgaris and Pohlia nutans with the constancy degree of V, there also occur: Hieracium pilosella, Solidago virgaurea, Peucedanum oreoselinum, Hypnum ericetorum, Festuca ovina, Vaccinium vitis-idaea, Pleurozium schreberi. In the Skwierzyna Forest, the association Pohlio-Callunetum usually covers relatively large areas and remains in the direct contact with a herb layer of pine forest Leucobryo-Pinetum, which is the final stage of its succession.

Seven objects were classified into this association by the authors of relevés. The set of relevés from the Dobrzyń Upland was called by Kępczyński (1965) as the community with Calluna vulgaris. However, despite the absence of Pohlia nutans, this group of relevés could also be classified within the nodding thread-moss heaths. It is characterized by a very poor species composition (column 1, Tab. 1). Despite the little documentary material (only 5 relevés), 
phytocoenoses from this area were classified within the association Pohlio-Callunetum due to the presence of three species characteristic of the alliance Pohlio-Callunion: $\mathrm{Di}$ cranum scoparium, common bent grass Agrostis vulgaris and cup lichen Cladonia pyxidata. It is distinguished, however, by the presence of certain species that do not occur either in the Skwierzyna Forest or on the artillery range near Torun. These are the following species: stag's-horn clubmoss Lycopodium clavatum, common woodrush $\mathrm{Lu}$ zula multiflora, heath rush Juncus squarrosus, common heathgrass Sieglingia decumbens, mat grass Nardus stricta and pill sedge Carex pilulifera. According to Kępczyński (1965), the succession of the community with Calluna vulgaris proceeds towards pine forests. A considerable contribution of coniferous forest species, such as: Scots pine Pinus sylvestris (the constancy degree of III), lingberry Vaccinium vitis-idaea (III), as well as cup lichens Cladonia furcata (II) and Cladonia arbuscula (V). From the floristic composition of the community with heather, described in the Dobrzyń Upland, it appears that some of the analysed patches significantly correspond to the so-called wet matgrass sward from the association Nardo-Juncetum squarrosi (All. Violion caninae, O. Nardetalia). This is confirmed by the presence of species such as mat-grass Nardus stricta and heath rush Juncus squarrosus. Also the contribution of peat-bog species indicates high moisture of the habitat - common sundew Drosera rotundifolia, grasslike sedge Carex panicea, common sedge $C$. nigra and few other. At the same time, the presence of coniferous forest species in this set of relevés indicates the impermanent nature of the community with Calluna vulgaris and a rather fast rate of the secondary succession process. The succession proceeds towards the transformation of heaths into pine forests, similarly like in the case of the association PohlioCallunetum in the Skwierzyna Forest and on the artillery range near Toruń.

The absence of the characteristic species Pohlia nutans is also noticeable in relevés made during the interwar period by Kobendza (1930) in the Kampinos Forest. However, on the floristic list and in the descriptions of communities of this forest complex, the author informs that the species often occurs on heaths. Whereas according to Kępczyński (1965), the community with Calluna vulgaris from the Dobrzyń Upland are very similar to communities with common heather from the Kampinos Forest, which were described by Kobendza (1930).

In order to compare the variability of ArctostaphyloCallunetum in Poland, the constancy coefficients for 115 species in 13 so far published sets of relevés of this association were compiled in Table 2. The first two columns present the association of bearberry heaths from north-eastern Poland (Faliński \& Bartel 1965), as well as from this part of Poland and from the neighbourhood of Leningrad in Russia (Tüxen 1942). They are characterized by the pres- ence of subboreal species, such as pasque flowers - Pulsatilla pratensis, Pulsatilla patens and Pulsatilla patens subsp. teklae, as well as bloody cranesbill Geranium sanguineum, devil's-bit scabious Succisa pratensis. Furthermore, in phytocoenoses of bearberry heaths in north-eastern Poland, close location of coniferous forests becomes apparent. It is manifested by the presence of coniferous forest species in all forest layers. These are the following species: Viola canina, Melampyrum pratense, Pinus sylvestris, Cladina arbuscula, Cladonia rangiferina, Pleurozium schreberi and Betula pendula. Whereas on bearberry heaths from the artillery range near Torun, pasque flowers were not recorded (a small pasque flower Pulsatilla pratensis is a very rare species on the training ground). Whereas the common feature of the described community from both regions is the occurrence of characteristic and differential species of the association, as well as some coniferous forest species.

Relevés in the fourth column present communities with Arctostaphylos uva-ursi from the Lublin Region, southeastern Poland. Among others, they are characterized by the presence of species from the class Koelerio glaucaeCorynephoretea canescentis: Corynephorus canescens, Polytrichum piliferum, as well as species from the class Vaccinio-Piceetea: Pinus sylvestris, Vaccinium vitis-idaea, Pleurozium schreberi, (the constancy degree of V). The author of relevés from the Lublin Region classified these communities within the central eastern variety of the association Spergulo-Corynephoretum. A common feature of their species composition with phytocoenoses from the artillery range near Torun is the occurrence of species of psammophilous grasslands.

Patches from the Skwierzyna Forest (column 5, Tab. 2) are distinguished by the presence of subatlantic species, such as: Pohlia nutans, Dicranum polysetum, Agrostis vinealis, Euphorbia cyparissias, Galium verum. Balcerkiewicz and Brzeg (1993) classified them within the CentralEuropean variant of the association, due to the presence of subatlantic species. The occurrence of Pohlia nutans proves the connection between bearberry heaths and nodding thread-moss heaths. Such a phenomenon is also observed on the artillery range near Torun.

Relevés from the Green Kurpie Forest (column 3, Tab. 2), as well as relevés from Opole Silesia not included in the Table, are not distinguished by any species. They are of intermediate character and correspond to both communities from north-eastern Poland, as well as heaths from the Lublin Region, south-eastern Poland.

The research by Ciosek (2000) in central-eastern Poland (123 relevés) revealed high internal heterogeneity of bearberry heaths. The author distinguished few subassociations, each of them characterized by the dissimilarity in species composition. 


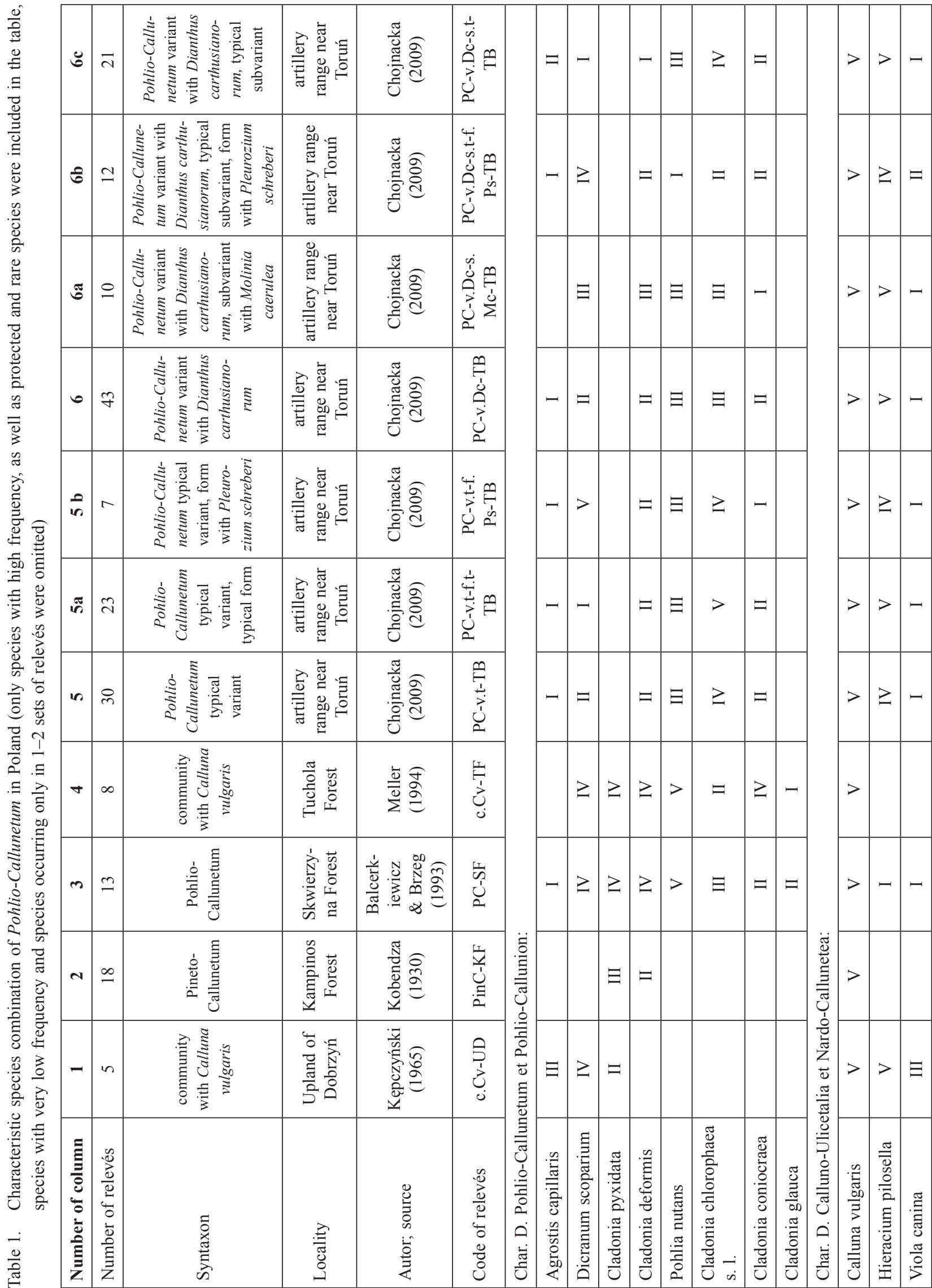




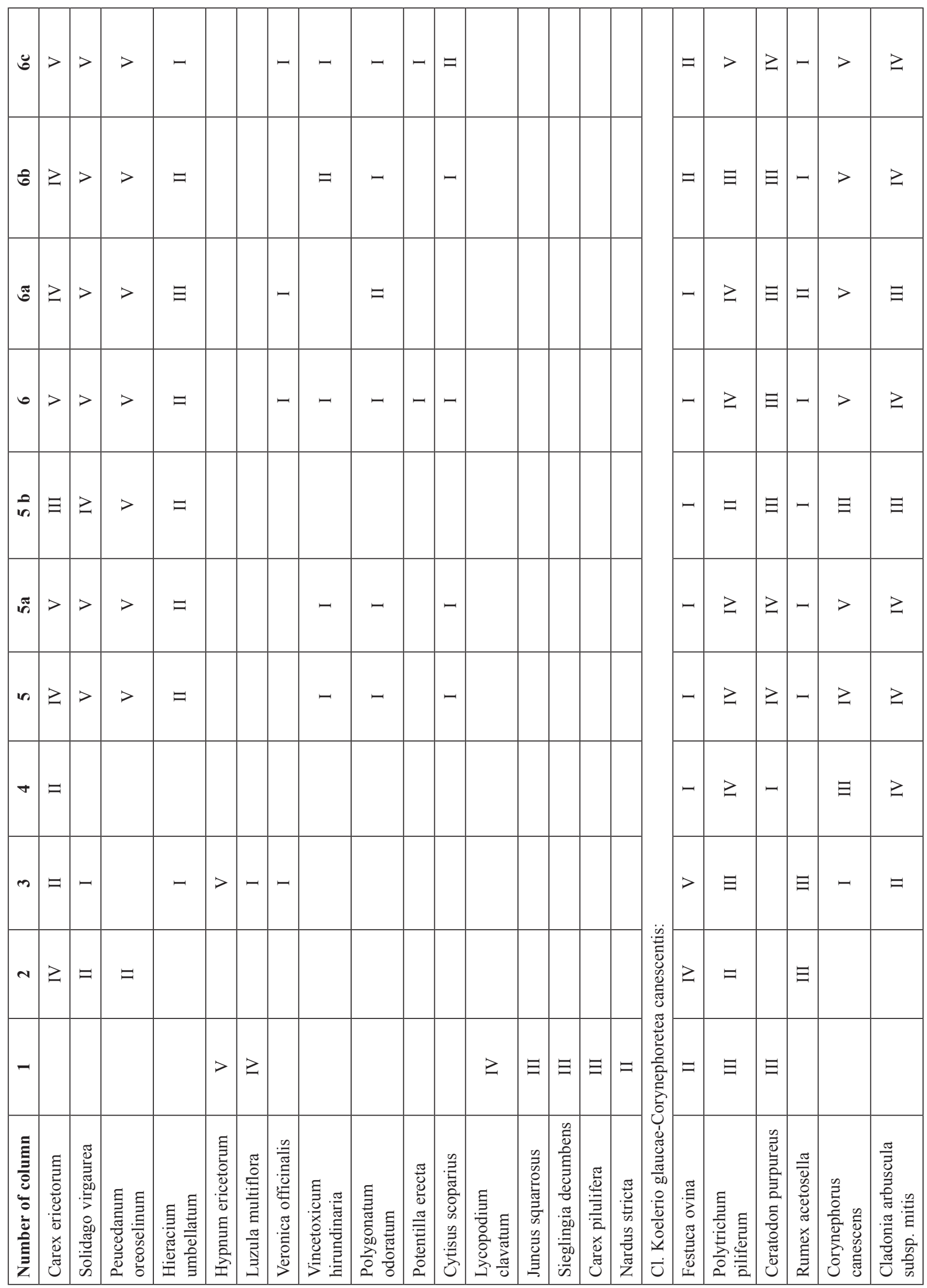




\begin{tabular}{|c|c|c|c|c|c|c|c|c|c|c|c|c|c|c|c|c|c|c|c|c|c|c|c|c|}
\hline 8 & - & $\Xi$ & $=$ & - & $=$ & $\geq$ & $\Xi$ & - & $=$ & - & $=$ & - & & & - & & & $\Xi$ & $z$ & $\Xi$ & $\neg$ & - & - & - \\
\hline ๑ & $=$ & $\Xi$ & & - & & $\geq$ & $\Xi$ & $=$ & - & $=$ & - & - & & $=$ & - & & & $\Xi$ & $\Xi$ & $\Xi$ & $\Xi$ & $=$ & $=$ & $=$ \\
\hline$\tilde{\sigma}$ & $\Rightarrow$ & $\Xi$ & & & & $\geq$ & $\Xi$ & - & - & - & -1 & & & - & & & & $=$ & $\Xi$ & $=$ & & - & $=$ & - \\
\hline 0 & $=$ & $\Xi$ & - & - & - & $\geq$ & $\Xi$ & - & $=$ & - & - & - & & - & - & & & $=$ & $\Xi$ & $\Xi$ & $=$ & - & $=$ & - \\
\hline in & - & $\Xi$ & & - & & $=$ & - & - & & $\Xi$ & & & & & & - & - & & & & & & - & $\exists$ \\
\hline దే & - & $\exists$ & - & & - & $=$ & $=$ & $\neg$ & - & - & - & - & & & & - & & & & & & - & - & - \\
\hline in & - & $\Xi$ & - & - & - & $=$ & $=$ & - & $\neg$ & - & -1 & - & & & & - & - & & & & & & - & - \\
\hline$\nabla$ & $\geq$ & $z$ & & $=$ & & & & & & & & & & & & & & & & & & & & \\
\hline$m$ & - & - & $\Xi$ & $=$ & - & & & & & & & & $\geq$ & & & & & & - & & & - & & $\geq$ \\
\hline$N$ & & & & & & & & & & & & & & & & & & & & & & & $\Xi$ & $\geq$ \\
\hline- & & & & & & & & & & & & & & & & & & & & & & & & $\Xi$ \\
\hline 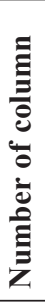 & 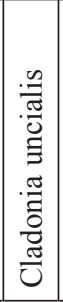 & 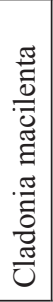 & 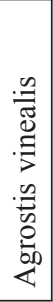 & 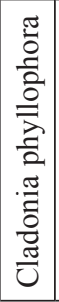 & 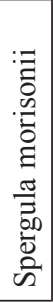 & 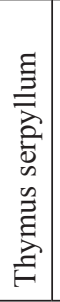 & 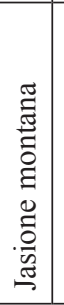 & 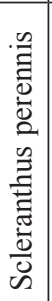 & 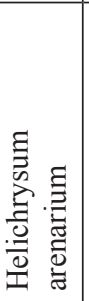 & 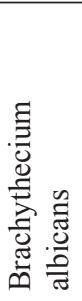 & 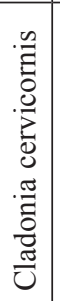 & 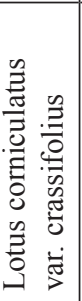 & 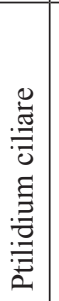 & 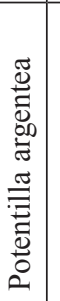 & 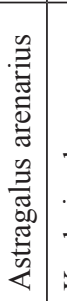 & 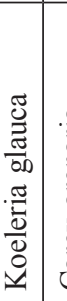 & 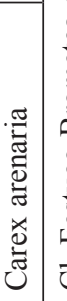 & 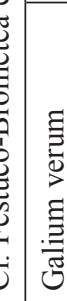 & 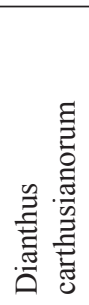 & 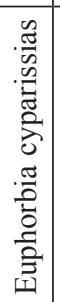 & 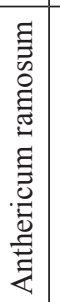 & 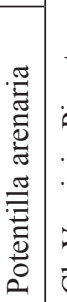 & 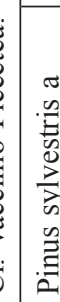 & 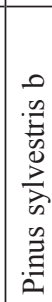 \\
\hline
\end{tabular}




\begin{tabular}{|c|c|c|c|c|c|c|c|c|c|c|c|c|c|c|c|c|c|c|c|c|c|c|c|c|}
\hline 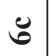 & $=$ & & $=$ & & & - & $=$ & $\Xi$ & $=$ & & & -1 & - & - & & & - & - & $\Xi$ & $\Xi$ & & & & \\
\hline 6 & $\Xi$ & $>$ & - & $\Xi$ & $=$ & $>$ & $=$ & $>$ & $\geq$ & & & $\Rightarrow=$ & $=$ & $=$ & & & -1 & - & - & $\Xi$ & & & & \\
\hline $\mathfrak{\Xi}$ & - & - & $=$ & & & - & $=$ & $\Xi$ & $=$ & & & $=1$ & -1 & $=$ & & & & $-1-$ & $\Xi$ & $\Xi$ & & $>$ & & \\
\hline 6 & $=$ & $=$ & $=$ & - & - & $=$ & $=$ & $\Xi$ & $=$ & & & $=1$ & $-1=$ & $=$ & & & -1. & $-1-$ & $=$ & $\Xi$ & & $=$ & & \\
\hline in & - & $>$ & - & $=$ & - & $>$ & $\Xi$ & $\geq$ & $=$ & & & $=1$ & $-1=$ & $=$ & & & & -1 & & - & & & & \\
\hline దే & $\Xi$ & & $=$ & - & - & - & $=$ & $\geq$ & $\Xi$ & & & $=\Rightarrow$ & $=$ & $=$ & & & - & $-1-$ & $\geq$ & - & - & & & \\
\hline in & $=$ & $=$ & - & - & - & $=$ & $=$ & $\geq$ & $\Xi$ & & & $\Rightarrow=$ & $=$ & $=$ & & & - & $-1-$ & $\Xi$ & - & - & & & \\
\hline+ & $z$ & $>$ & - & $>$ & & $\geq$ & $\geq$ & $>$ & $>$ & - & & & & & & & & $=$ & & & & - & - & $\Xi$ \\
\hline$\infty$ & $\geq$ & $>$ & $\Xi$ & $\Xi$ & $>$ & $\geq$ & - & $\Xi$ & $\equiv$ & $=$ & & & -1 & - & & & & & $=$ & & & - & - & - \\
\hline$\sim$ & & $\geq$ & & & & $\geq$ & $>$ & & & & & $=-$ & - & & & $-1=$ & $=$ & $E$ & & & & & $z$ & \\
\hline- & & $>$ & $=$ & $>$ & $\Xi$ & & & & & & & & $=$ & & $=$ & & - & $=$ & $>$ & & $\Xi$ & - & $=$ & $=$ \\
\hline 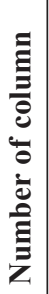 & 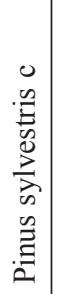 & 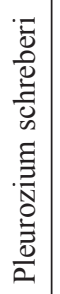 & $\begin{array}{l}\underset{\frac{\pi}{0}}{0} \\
\stackrel{0}{2} \\
. \frac{\pi}{3} \\
\frac{0}{0} \\
\frac{\pi}{U}\end{array}$ & 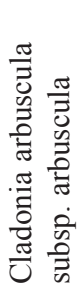 & 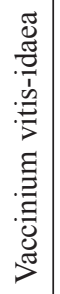 & 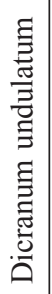 & 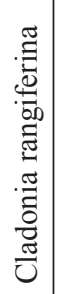 & 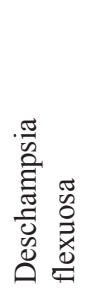 & 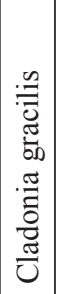 & 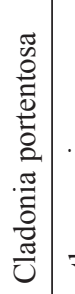 & 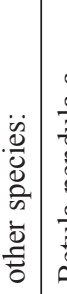 & 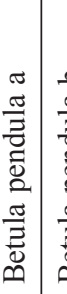 & 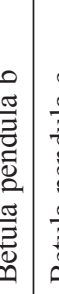 & 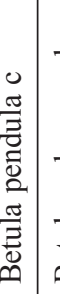 & 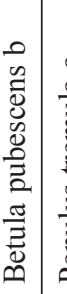 & 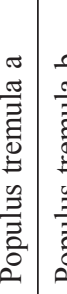 & 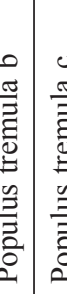 & 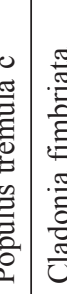 & 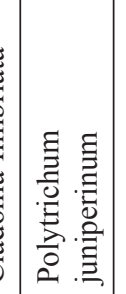 & 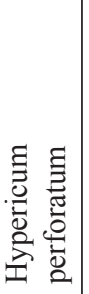 & 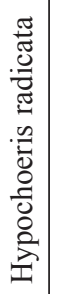 & 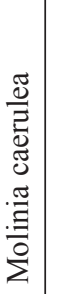 & 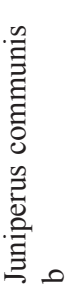 & 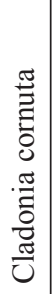 \\
\hline
\end{tabular}




\begin{tabular}{|c|c|c|c|c|c|c|c|c|c|c|c|c|c|c|c|c|c|c|c|c|c|c|c|}
\hline $\mathscr{8}$ & & $>$ & & & & & & & & - & - & & & & & & & & & & & & \\
\hline 8 & & $\geq$ & $\exists$ & & & & & & & - & - & & & & & & & & & & & & \\
\hline $\mathbb{\sigma}$ & & $>$ & & & & & & & - & - & & & & & & & & & & & & & \\
\hline 6 & & $\geq$ & - & & & & & & - & - & - & & & & & & & & & & & & \\
\hline in & & $\Xi$ & $>$ & & & & & & - & - & $\exists$ & & & & & & & & & & & & \\
\hline ถ็ & & $>$ & & & & & & & - & & - & & & & & & & & & & & & \\
\hline in & & $\geq$ & - & & & & & & - & - & $=$ & & & & & & & & & & & & \\
\hline$\nabla$ & $=$ & & - & $\geq$ & $\exists$ & $=$ & $=$ & $\Rightarrow$ & $>$ & $\geq$ & & & & & & & & & & $=$ & $=$ & $=$ & $=$ \\
\hline$m$ & & $\Xi$ & - & - & - & - & - & - & & & & & & - & $=$ & & & & & & & & \\
\hline 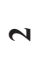 & $\Xi$ & & & & & & & & & & & - & $=$ & & & & & $\Xi$ & $=$ & & & & \\
\hline- & & & & & & & & & & & & & & & & $=$ & $=$ & & & & & & \\
\hline 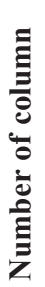 & 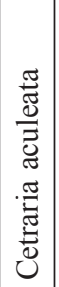 & 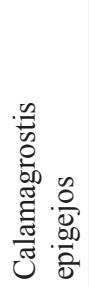 & 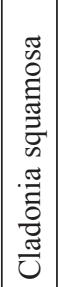 & 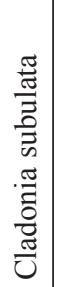 & 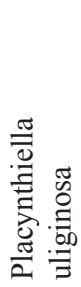 & 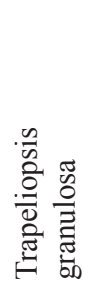 & 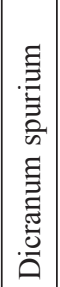 & 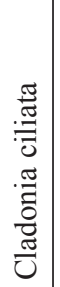 & 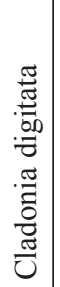 & 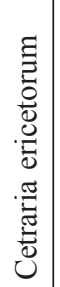 & 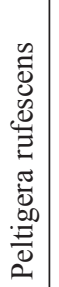 & 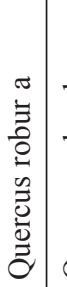 & $\begin{array}{l}0 \\
\stackrel{\Xi}{0} \\
0 \\
0 \\
0 \\
0 \\
0 \\
0 \\
0 \\
\overrightarrow{0}\end{array}$ & 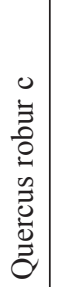 & 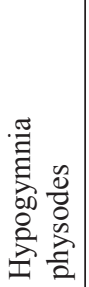 & 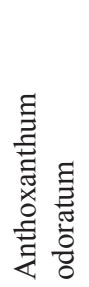 & 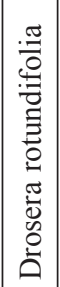 & 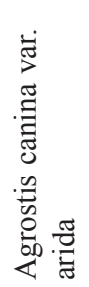 & 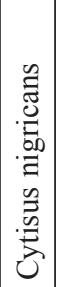 & 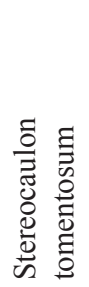 & 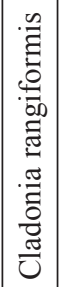 & 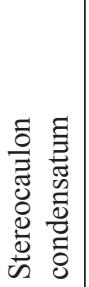 & 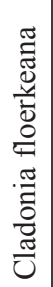 \\
\hline
\end{tabular}




\begin{tabular}{|c|c|c|c|c|c|c|c|c|c|c|c|c|c|c|c|c|c|c|c|}
\hline 3 & 6 & 妾 & 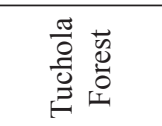 & 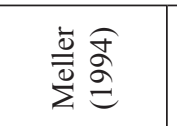 & 崫 & & $>$ & - & & & & & $z$ & - & & & & & \\
\hline$\approx$ & ते & 妾 & 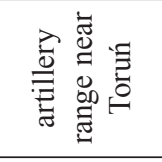 & 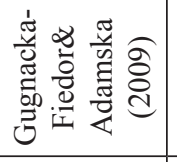 & 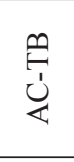 & & $\geq$ & $\geq$ & $\geq$ & & $\Xi$ & & $>$ & $=$ & - & - & & & \\
\hline$=$ & 0 & 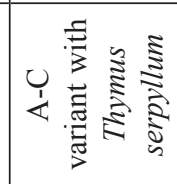 & 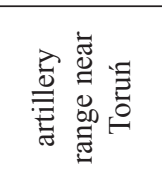 & 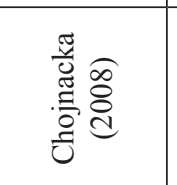 & 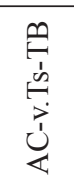 & & $>$ & $\Xi$ & $>$ & $>$ & -7 & $\geq$ & $>$ & & $=$ & $=$ & & & \\
\hline$\varrho$ & in & 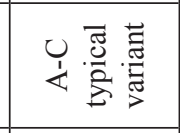 & 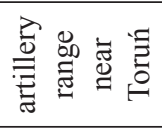 & 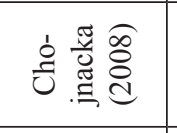 & 䇋 9 & & $>$ & $>$ & $>$ & $>$ & & & $>$ & & & & & & \\
\hline$a$ & $\stackrel{i}{m}$ & 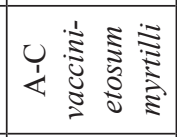 & 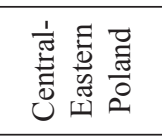 & 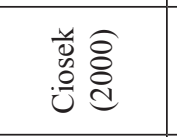 & 兽 & & $>$ & $\Xi$ & $=$ & $>$ & $=$ & & $>$ & $=$ & - & - & - & & $\Xi$ \\
\hline$\infty$ & $m$ & 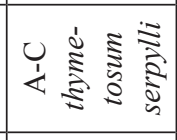 & 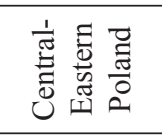 & 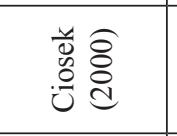 & 穿出 & & $>$ & $\geq$ & $=$ & $=$ & - & & $>$ & - & $=$ & & $\geq$ & -1 & - \\
\hline$r$ & ते & 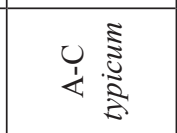 & 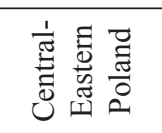 & 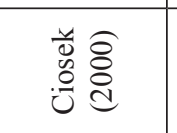 & 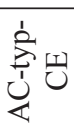 & & $>$ & $\Xi$ & & $=$ & & & $>$ & & & & - & & - \\
\hline o & $\approx$ & 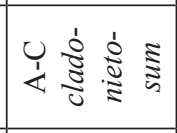 & 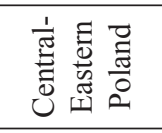 & 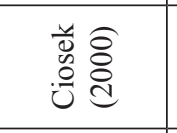 & 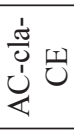 & & $>$ & $\Xi$ & - & - & & & $>$ & - & & - & & - & - \\
\hline in & in & 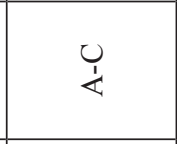 & 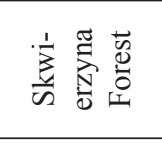 & 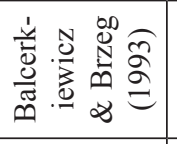 & 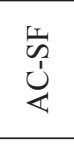 & & $\Xi$ & $>$ & - & & - & & $>$ & & $\Xi$ & - & & & \\
\hline+ & 은 & 主 & 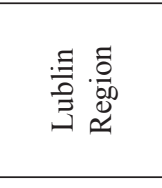 & 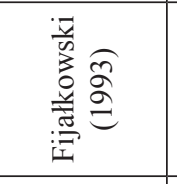 & 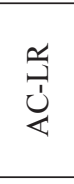 & & $>$ & $\neg$ & - & & & & $>$ & & & - & - & - & - \\
\hline$m$ & 0 & 变 & 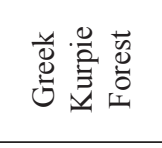 & 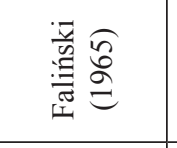 & 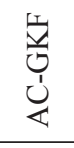 & & $>$ & $=$ & - & & & & $>$ & - & & - & & - & \\
\hline N & $r$ & 岁 & 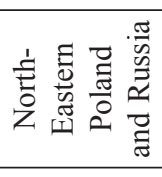 & 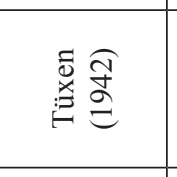 & 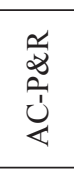 & & $>$ & $>$ & $=$ & $=$ & & $\frac{\pi}{0}$ & $>$ & & $\Xi$ & - & - & $=$ & - \\
\hline- & $\infty$ & 峦 & 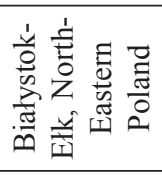 & 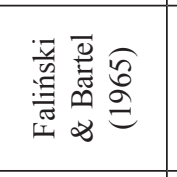 & 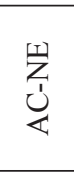 & ₹ี & $>$ & $>$ & $\geq$ & $z$ & & & $>$ & & $\Xi-$ & -- & $z$ & $=$ & \\
\hline 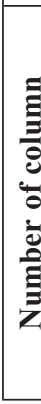 & 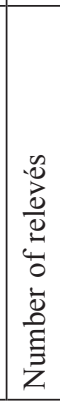 & 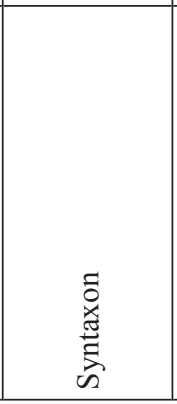 & 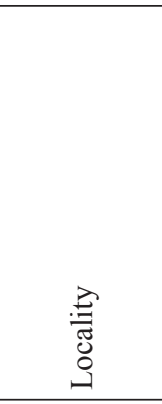 & 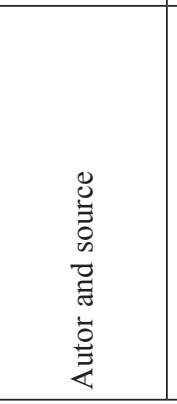 & 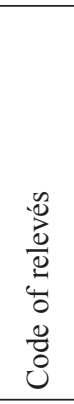 & 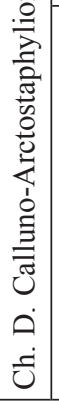 & 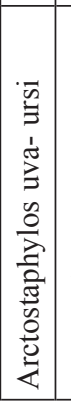 & 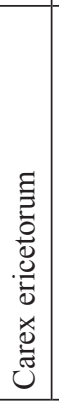 & 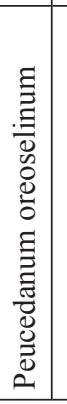 & 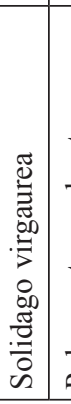 & 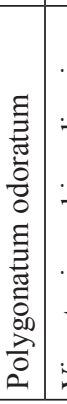 & 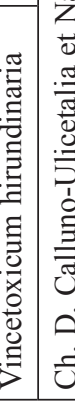 & 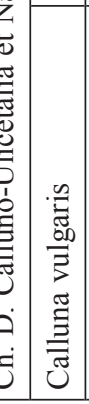 & 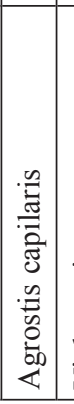 & 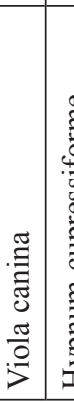 & 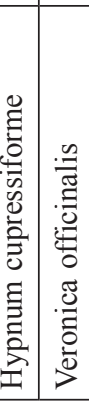 & 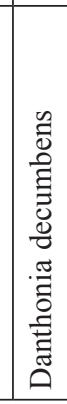 & 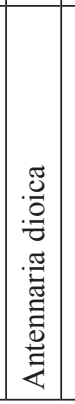 & 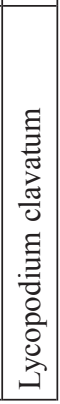 \\
\hline
\end{tabular}




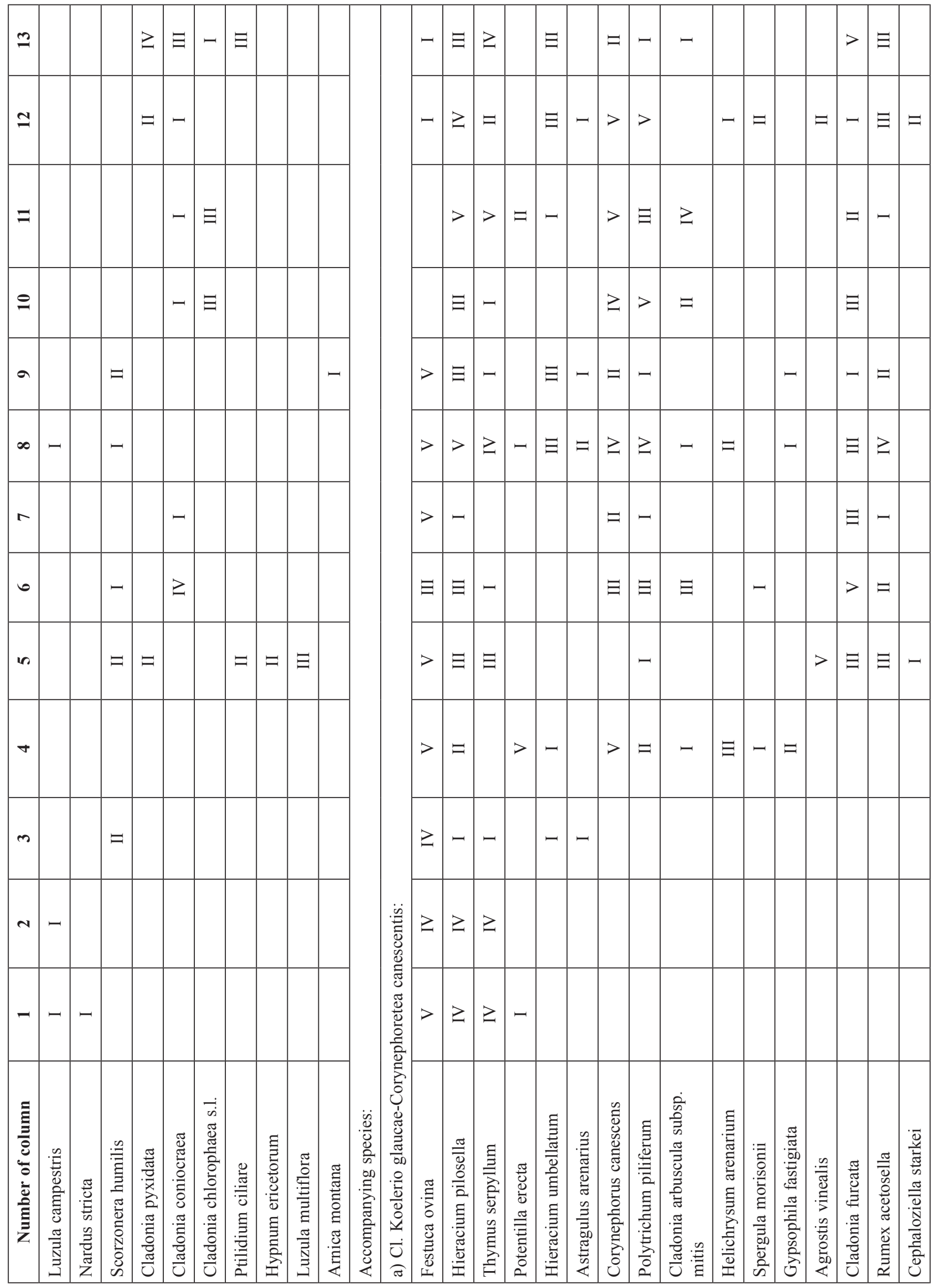




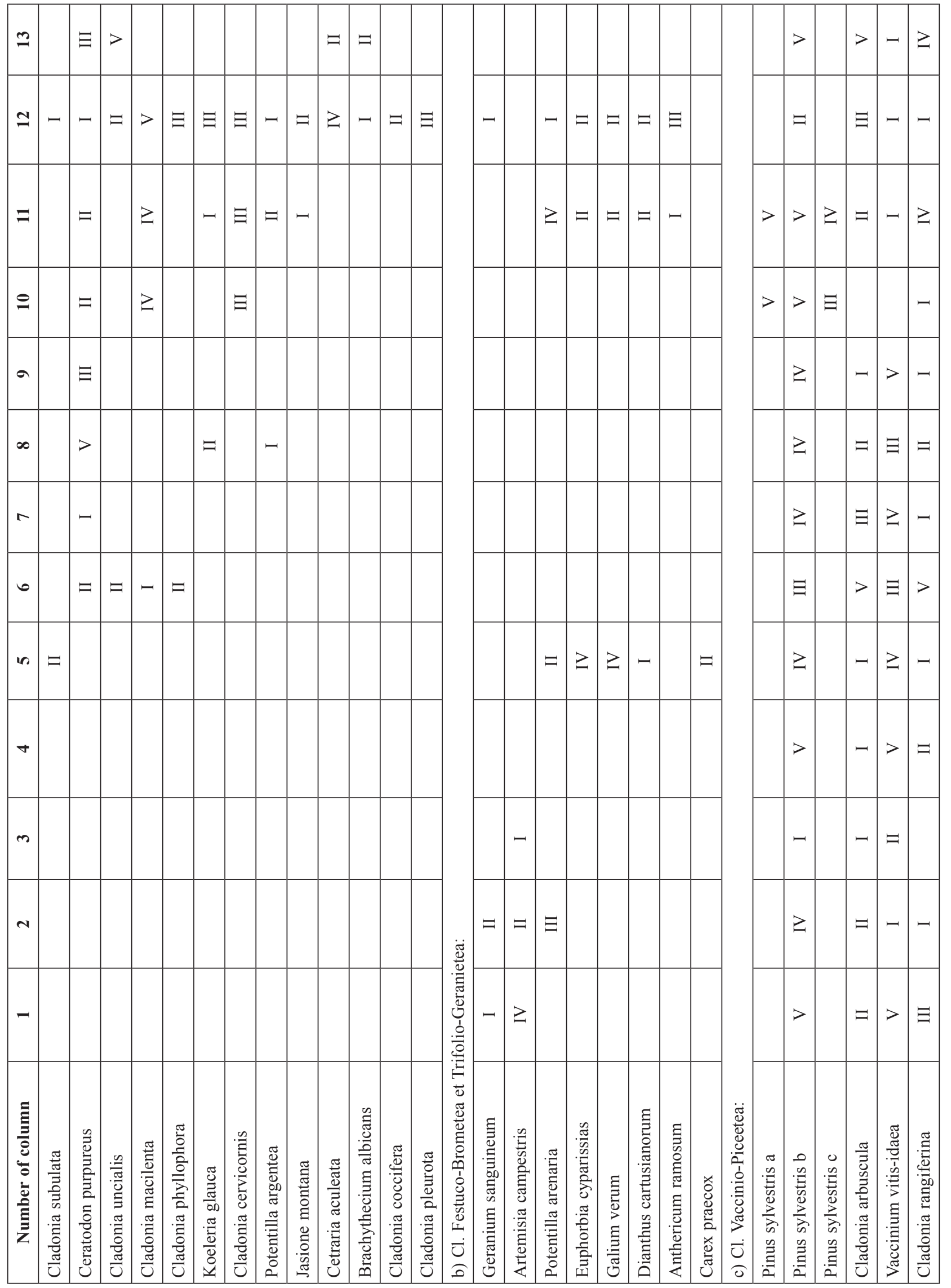




\begin{tabular}{|c|c|c|c|c|c|c|c|c|c|c|c|c|c|c|c|c|c|c|c|c|c|c|c|c|c|c|c|}
\hline$\cong$ & $>$ & $\geq$ & & & $\geq$ & $>$ & & & $>$ & & & & & & & & & & & & & & & - & - & & \\
\hline$\approx$ & - & & - & & - & $=$ & & & $=$ & & & & & & & - & & & & & $\exists-$ & - & & $=$ & - & -1 & $=$ \\
\hline$=$ & $=$ & $\geq$ & & $=$ & $\exists$ & $\geq$ & & & & & & & & & & $=$ & & & & & $>$ & & $=$ & $=$ & & & \\
\hline$\varrho$ & & & & & & & & & & & & & & & & & & & & & $\exists$ & & $=$ & $=$ & & & \\
\hline$a$ & $>$ & $>$ & $>$ & $z$ & - & & & & & $\Xi$ & - & - & - & & & & & & & & -1 & - & & $=$ & - & $z$ & - \\
\hline$\infty$ & $\geq$ & $\geq$ & $z$ & - & $=$ & - & - & & & & & & & & & - & & & & & $-1=$ & $=$ & & - & - & $=$ & \\
\hline$r$ & $\geq$ & $\geq$ & $\geq$ & - & - & & & & & -1 & - & $=$ & & & & & & - & & & & - & & - & & $=$ & \\
\hline 0 & $z$ & $\geq$ & $\geq$ & - & & & & & $\Xi$ & - & & - & & & & & & & & & - & & & - & - & $\exists$ & - \\
\hline in & $>$ & $>$ & & - & $\exists$ & - & $=$ & $=$ & & & & & & & & $=$ & & $\Xi$ & $=$ & & $>$ & & & & & & \\
\hline$\sigma$ & $>$ & $=$ & & $>$ & & & & & & & & & & & & & & & & & & & $\exists$ & & & & \\
\hline m & - & - & $=$ & & & & & & & & & & & & & & & & & & - & & & & & - & - \\
\hline$N$ & $\exists$ & - & - & & & & & & & & & & & & & $\Xi$ & - & & & & & & - & & - & $\exists$ & - \\
\hline- & - & - & $\geq$ & & & & & & & & & & $\geq$ & - & & $=$ & $=$ & & & & & & - & & $\exists$ & $\geq$ & $\exists$ \\
\hline 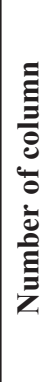 & 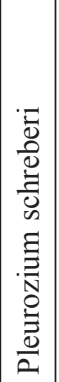 & 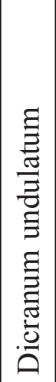 & 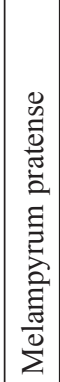 & 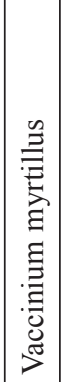 & 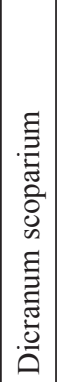 & 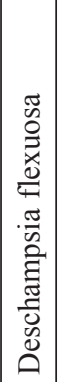 & 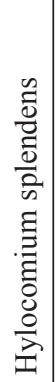 & 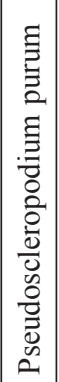 & 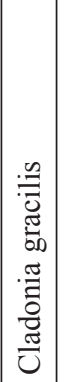 & 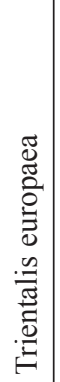 & 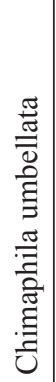 & 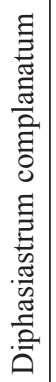 & 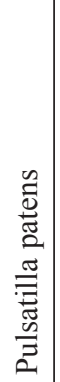 & 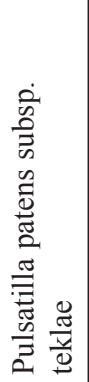 & 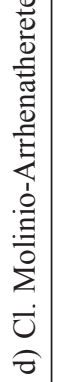 & 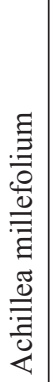 & 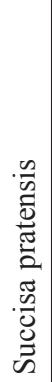 & 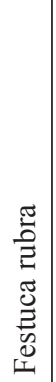 & 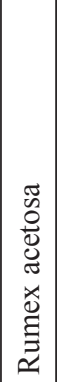 & 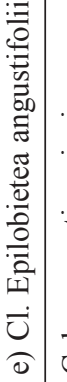 & 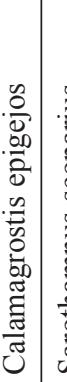 & 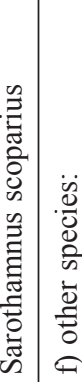 & 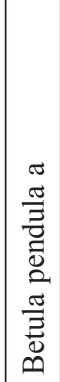 & 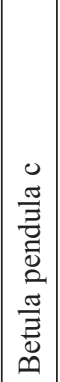 & 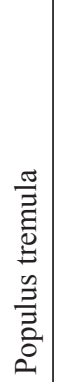 & 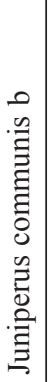 & 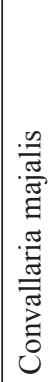 \\
\hline
\end{tabular}




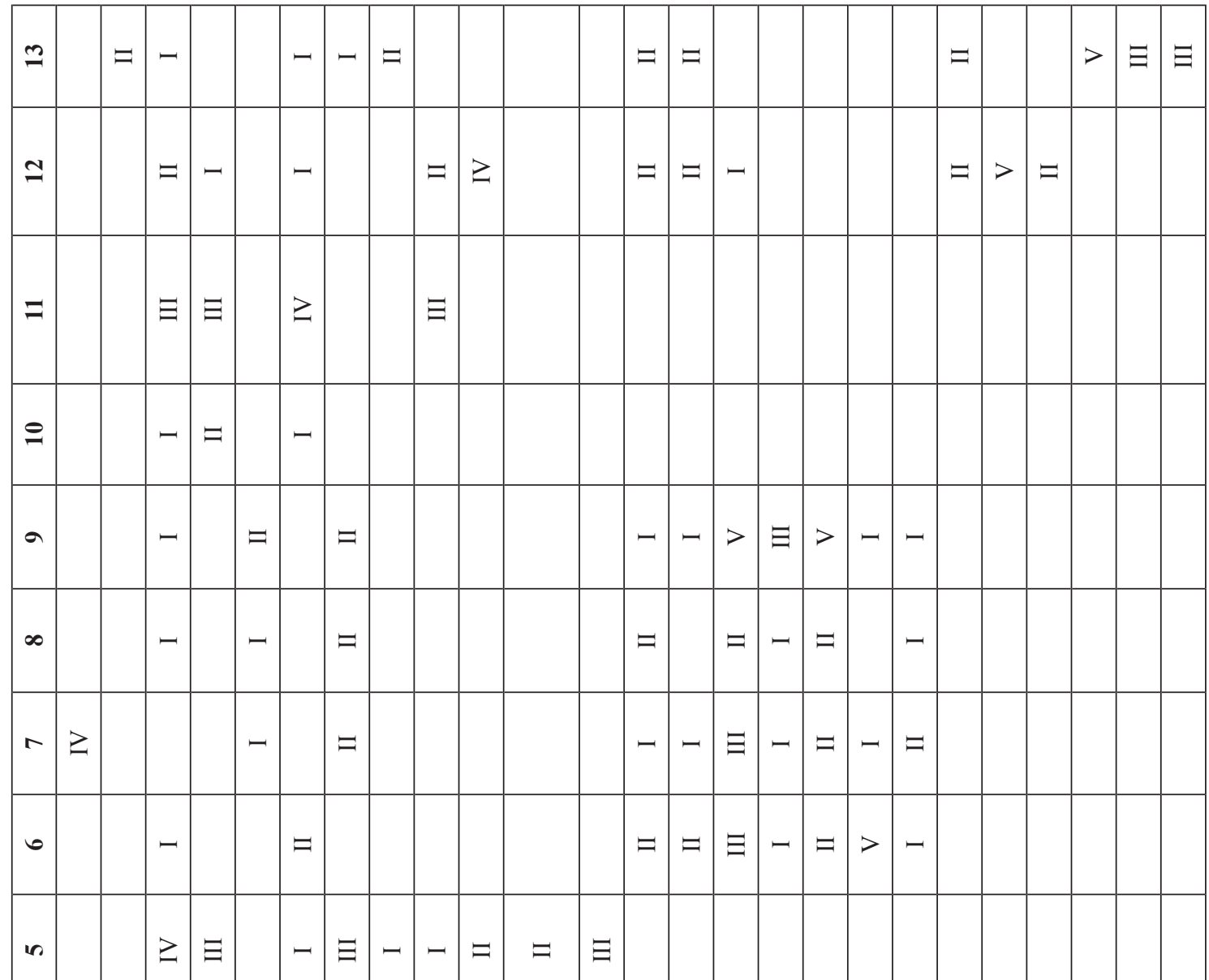

$+\quad \exists->=$

$m$

$\sim=$

$-=$

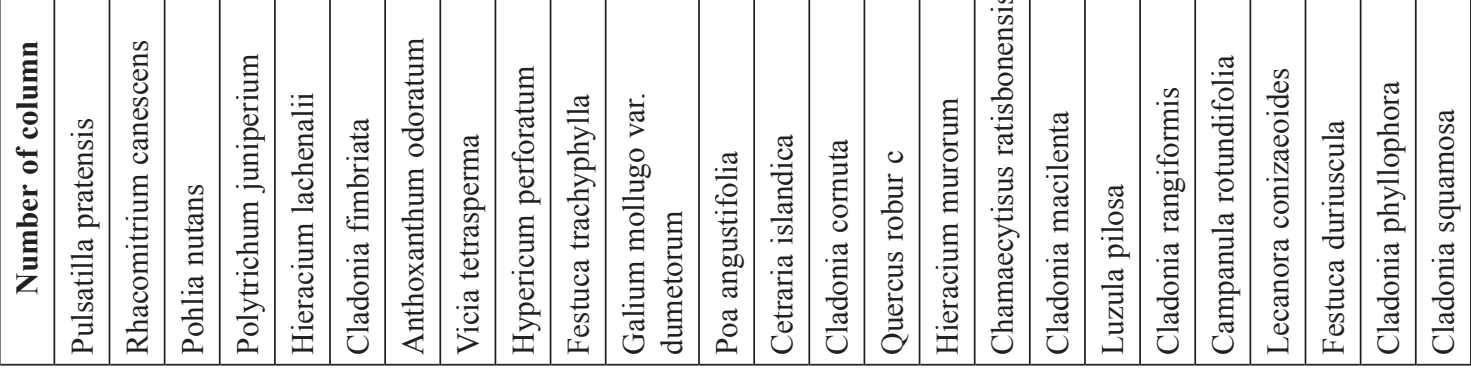


Analysis of the floristic composition of bearberry heaths from central-eastern Poland (columns 6-9, Tab. 2) and the vicinity of Torun (columns 10-12, Tab. 2) reveals that patches of the thyme subassociation and the subassociation vaccinietosum myrtilli (central-eastern Poland) have most of the characteristics common with the bearberry heath from the artillery range. At both locations, species from pine forests encroach on bearberry heaths, which indicates the progressing secondary succession towards pine forests. Also relevés of bearberry heaths from the Tuchola Forest (column 13, Table 2) are quite similar to relevés from the artillery range near Torun.

The contribution of kinnikinnick bearberry in relevés from Zadroże dunes, near the city of Torun, which were not included in Table 2, is several times smaller as compared to common heather. This resembles them to some of the patches of bearberry heaths from the training ground, which gradually transform into forest communities.

\subsection{Syntaxonomic analysis with the software MVSP}

In the dendrogram drawn up with the minimum variance method, and using the squared Euclidean distance as a measure of distance, Pohlio-Callunetum heaths from the artillery range near Torun are evidently separated from the others. The sets of relevés from the vicinity of Torun comprising five syntaxa of a lower rank, i.e. subassociations, variants and forms, form one cluster (Fig. 1A). There is a considerable similarity between these sets, as fusions occur at low levels corresponding to small Euclidean distances. The other four sets of relevés, made in other regions of Poland, form a separate cluster within which the distances between particular objects are significant (Fig. 1A). The smallest distance in this group of objects occurs between heaths from the Skwierzyna Forest and from the Tuchola Forest.

In the dendrogram obtained with the median method, using the square of the Gower's General Similarity Coefficient, both forms are very similar to each other, i.e. the typical one and the one with Pleurozium schreberi of the typical variant from the artillery range near Torun (Fig. 1B). There is a great similarity between the subsets of these relevés and the subvariant with Pleurozoium schreberii of the variant with Dianthus carthusianorum from the same area. The two other sets of relevés, representing the variant with Dianthus carthusianorum from the artillery range near Torun, form a separate cluster. Their similarity is significant.

In the dendrogram drawn up with the median method also a high similarity between heaths from the Dobrzyń Upland and the Kampinos Forest is evident, which is in accordance with the statement of Kępczyński (1965) that the species composition of moorlands from these regions is similar. In all objects of this cluster, species from pine forests are relatively significant, particularly the Scots pine. At a higher level of fusion, the sets of relevés from these two regions form a cluster common with the sets of relevés from Skwierzyna Forest and Tuchola Forest (Fig. 1B). The similarity between these two subsets of relevés is smaller than between the relevés from the Dobrzyń Upland and the Kampinos Forest. In the analysis carried out with the minimum variance method, the coefficients of similarity between these subsets of relevés had the opposite pattern, i.e. the similarity between the relevés from the Skwierzyna Forest and the Tuchola Forest was higher than between the relevés from the Dobrzyń Upland and the Kampinos Forest (Fig. 1A).

In the dendrogram drawn up with the UPGMA method, applying the percentage similarity (Fig. 1C), all five sets of relevés from the training ground near Torun form one cluster. However at a high level of fusion, the sets of relevés from Skwierzyna Forest and Tuchola Forest demonstrate the highest similarity with the aforementioned cluster. The sets of relevés from the Dobrzyń Upland and from the Kampinos Forest differ significantly from the sets of this cluster (Fig. 1C).

In the numerical classification of the sets of Arctostaphylo-Callunetum relevés, carried out with the minimum variance method, applying the Squared Euclidean Distance, the association Arctostaphylo-Callunetum from the artillery range near Torun is clearly different from the sets of relevés from bearberry heaths from other regions of Poland. At the same time, both variants from the artillery range, i.e. the typical one and the one with Thymus serpyllum are very similar to each other. Whereas, the similarity between the discussed variants with the relevés made about ten years ago on the artillery range near Torun by Gugancka-Fiedor and Adamska (2010) is not particularly high. The cluster created by the tables of these three sets of relevés from the military area, groups together with other clusters at the highest level of fusion, corresponding to a considerable distance between the merged groups (Fig. 2A).

If the median method and the Gower's General Similarity Coefficient are applied to obtain a dendrogram, the three sets of relevés from the training ground near Torun display a relatively high similarity with the tables of Arctostaphylo-Callunetum from Skwierzyna and Tuchola Forests (Fig. 2B).

In both classifications, i.e. performed with the minimum variance method and with the median method, there is only a small similarity between the variant with Thymus serpyllum of association Arctostaphylo-Callunetum from the artillery range near Torun and the subassociation Arctostaphylo-Callunetum thymetosum serpylli from central-eastern Poland distinguished by Ciosek (2000). In the dendrogram drawn up with the minimum variance method and the Squared Euclidean Distance (Fig. 2A), four subassociations described by Ciosek (2000) form one cluster. In the dendrogram drawn up with the median method using the Gower's General Similarity Coefficient (Fig. 2B), there is a fusion, 
however on a low level of similarity, between four subassociations from central-eastern Poland and phytocoenoses from the Lublin Region described by Fijałkowski (1993), as well as from the Kurpie Forest described by Faliński (1965) and other regions in the central and north-eastern part of Poland and from Russia described by various authors. In the dendrogram drawn up with the minimum variance method, the highest similarity occurs between the subassociations vaccinietosum myrtilli and typicum from central-eastern Poland (Fig. 2A), whereas in the analysis performed with the median method, the highest similarity occurs between the subassociations vaccinietosum myrtilli and thymetosum serpylli from this part of Poland (Fig. 2B).

In the dendrogram drawn up with the UPGMA method, using the Percent Similarity (Fig. 2C), the set of relevés from the Tuchola Forest is the most distinguishable one. Three sets of relevés from the training ground near Torun form a separate cluster, which reveals low similarity with the sets of relevés from other regions of Poland. The sets of relevés from north-eastern Poland and from Russia, as well as the sets of relevés made by Ciosek (2000) in central-eastern Poland are characterized by high similarity. In the case of sets of relevés from the latter region, similarly like in the analyses performed with the minimum variance method, high similarity is revealed by pair of sets (i.e. relevés of subassociations) vaccinietosum myrtilli and typicum (Fig. 2C).

\section{Discussion and conclusions}

From the phytosociological analysis presented above and analyses included in other studies (e.g. Chojnacka et al. 2009) on the association of nodding thread-moss heaths Pohlio-Callunetum from different locations of Poland, it appears that it is an impermanent community, the species composition of which includes both elements of pioneer Corynephorus grasslands, as well as of pine forests, which are recognized as the final effect of secondary succession. Depending on the neighbouring plant communities, species composition and directions of succession transformations tend to change.

The analysis of all so-far published phytosociological studies concerning bearberry heaths Arctostaphylo-Callunetum in Poland, confirms the thesis of Ciosek (2000) that this association, similarly like Pohlio-Callunetum, is an impermanent community, threatened by displacement of the dominant and characteristic species, i.e. Arctostaphylos uva-ursi, mainly by pine forest species and common heather. Together with the increasing density of the canopy of trees and competition by heath plants, conditions for the development of bearberry become more difficult; also the structure and the size of clumps change. Bearberry ceases to form species poor aggregations. In the place of patches with the area of few square metres, small clusters are being formed. Bearberry becomes one of the species from the forest herb layer.

In the numerical analyses, the similarity between the sets of relevés with a very changeable species combination was studied. Species composition in some of the sets corresponded to syntaxa, which could not be classified as typical heaths. Such sets were included mainly in the analyses dealing with bearberry heaths Arctostaphylo-Callunetum. Some relevés in the set from the Lublin Region corresponded to psammophilous grasslands or pine forests with a high contribution of Arctostaphylos uva-ursi in the herb layer. Whereas in the analyses on the nodding thread-moss heaths Pohlio-Callunetum the sets of relevés without e.g. characteristic species Pohla nutans were used. These were relevés by Kępczyński (1965) from the Dobrzyń Upland and by Kobendza (1930) from the Kampinos Forest. Both sets comprised quite a lot of species from the class $\mathrm{Vac}$ cinio-Piceetea and corresponded to associations of fresh pine forest with a high contribution of Calluna vulgaris in the herb layer. Including this kind of relevés in numerical analyses, in order to increase the small number of relevés made so far on Polish heathlands, caused that indices of similarity between the objects in the obtained dendrograms often had low values.

In the numerical analyses, similarities and differences were studied between the sets of relevés within two syntaxa, i.e. Pohlio-Callunetum and Arctostaphylo-Callunetum. Similarities between relevés classified, according to the classic phytosociological method of Braun-Blanquet, into two distinct syntaxa, were not investigated. However, the distance or similarity between relevés from both groups, calculated by numerical taxonomy methods, could be considerable. This remark particularly concerns the set of relevés from the association Arctostaphylo-Callunetum, the variant with Thymus serpyllum from the training ground near the city of Torun. The constancy coefficient of III obtained by the species Pohlia nutans in this set of relevés and the lower cover (from + to 2) of Arctostaphylos uva ursi, could bring about the fact that, through the species composition and abundance, these relevés correspond to the nodding thread-moss heaths Pohlio-Callunetum. To prove this assumption, an additional numerical analysis is required on the set of relevés classified into both heath associations.

In the performed numerical analysis, degrees of constancy were used as characteristics of the objects. The additional information included in the cover coefficients was not taken into consideration. Moreover, the sets of relevés were not equally numerous, which could also affect the values of the similarity or distance coefficients, and consequently the connections between the objects in the obtained dendrograms.

Furthermore, phytosociological tables were accepted as objects in the performed analysis. Similarities between 
A.

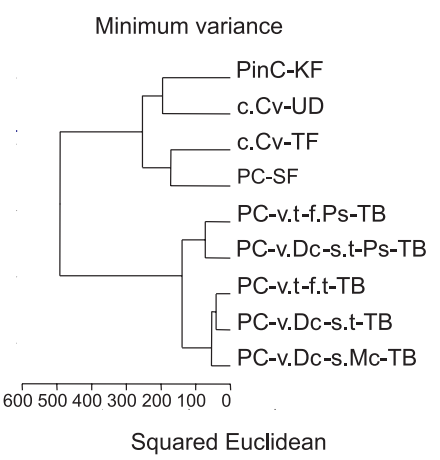

B.

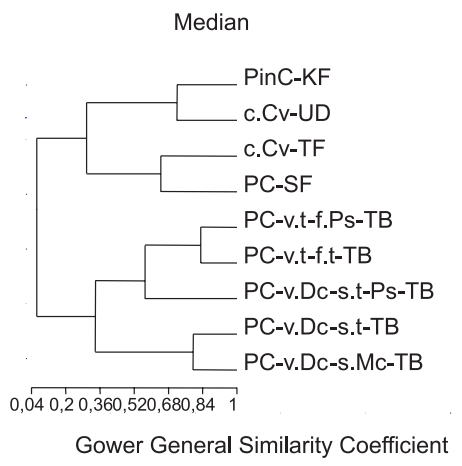

C.

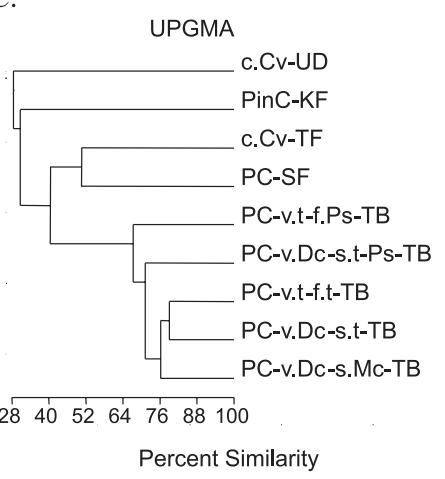

Figure 1. Dendrograms constructed with the minimum variance method (A), median method (B) and UPGMA (C), for 9 sets of relevés made in Pohlio-Callunetum and in similar plant communities (symbols of objects provided in the text and in Tab. 1)

A.

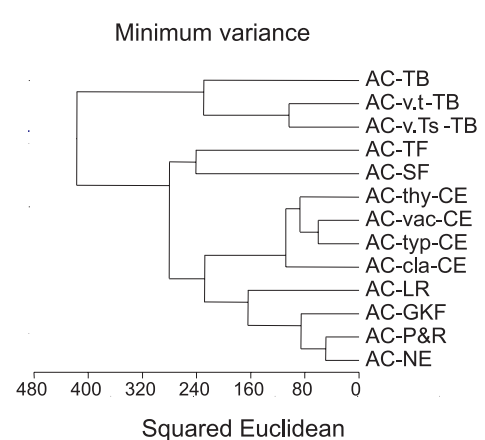

B.

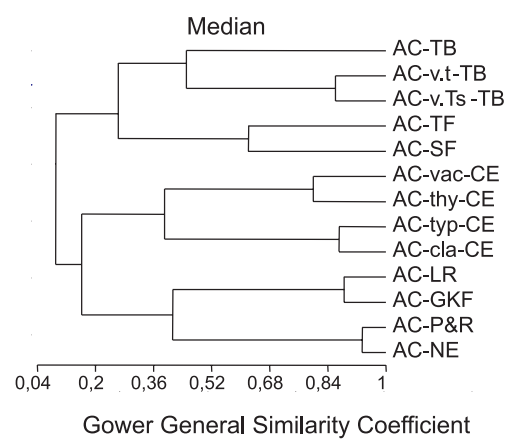

C.

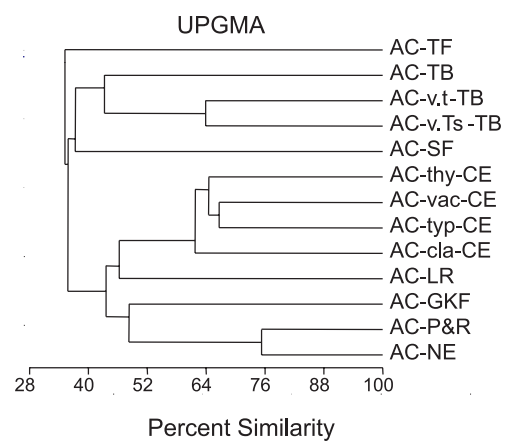

Figure 2. Dendrograms constructed with the minimum variance method (A), median method (B) and UPGMA (C), for 9 sets of relevés made in Arctostaphylo-Callunetum and in similar plant communities (symbols of objects provided in the text and in Tab. 2)

individual relevés included in a table were not investigated with the numerical methods. Perhaps one should also check the similarities between relevés of both heath associations. As it may happen that the similarity between relevés from different plant associations will be higher than between relevés of the same plant association.

Based on the analysis of tables and figures included in this work, one can reach a conclusion that the similarity between sets of relevés and, at the same time, the structure of dendrograms, were also affected by the fact that the relevés were made by different persons. Probably for this reason, the considerable differences, e.g. between relevés made by different persons on the artillery range near Torun, were evident in the obtained dendrograms. Also the fact that the relevés were made at the time interval of ca. 10 years, could have some influence on the significant differences between these sets of relevés.

Based on the performed numerical analyses, relevés from the artillery range near Torun were found considerably different from relevés of the similar syntaxa from other regions of Poland. The difference is evident even between the similar syntaxa of a lower rank, e.g. between the sets of relevés representing the variant with Thymus serpyllum from the training ground near Torun and the subassociation Arctostaphylo-Callunetum thymetosum serpylli from central-eastern Poland. Perhaps this is affected by the specific land use in the conditions of military training ground, as well as by the transformations of non-forest heath communities into forest communities taking place during the last years. Clarification of these causes requires further observations, mainly on the succession of heathlands.

\section{References}

Anikiejówna Cz. \& Gorska J., 1949, Stosunki florystyczne i fitosocjologiczne wydmy Zadroże [Floristic and phytosociological connections of the dune Zadroże], [in:] J. Prüffer (ed.) Z badań zespołowych wydmy Zadroże pod Toruniem [Collective investigations concerning 
the Zadroże dune near Toruń], Studia Societatis Scientiarum Torunensis, Supplementum 1, Torun - Polonia: $3-10$.

Balcerkiweicz S.\& BrzegA., 1993, Wrzosowiska przydrożne w kompleksie leśnym Borów Skwierzyńskich [Heath formations accompanying roads in the forest complex Bory Skwierzyńskie], Badania Fizjograficzne nad Polską Zachodnią, Ser. B, 42 :105-127.

Braun-Blanquet J., Sissingh C. \& Vlieger J., 1939, Klasse der Vaccinio-Piceetea, Prodromus der Pflanzengesellschaften 6, Montpellier.

Chojnacka J., 2009, Murawy napiaskowe i wrzosowiska na poligonie artyleryjskim pod Toruniem - trwałe zbiorowiska, czy pionierskie stadia lasu? [Psammophilous grasslands and heaths on the artillery range near the city of Torun - permanent communities, or pioneer forest phases?], Master Thesis, Faculty of Biology and Earth Sciences, Dept. of Plant Ecology and Nature Protection, NCU, Torun.

Chojnacka J., Cyzman W., Nienartowicz A. \& Deptuła M., 2010, Variability of the structure and directions in the development of heaths and arenaceous grasslands within the artillery range near the city of Torun, Ecological Questions 12 (this issue).

Ciosek M.T., 2000, Mącznica lekarska Arctostaphylos uva-ursi i wrzosowiska mącznicowe ArctostaphyloCallunetum w Polsce środkowowschodniej na tle ich zróżnicowania w Polsce [Bearberry, Arctostaphylos uva-ursi (L.) Spring., and bearberry moors, Arctostaphylo-Callunetum R. Tx. et Prsg 1949, in eastern central Poland in relation to their diversity in Poland], Rozprawa Naukowa Nr 65, Wydawnictwo Akademii Podlaskiej, Siedlce.

Faliński J. B., 1965, O roślinności Zielonej Puszczy Kurpiowskiej na tle stosunków geobotanicznych tzw. Działu Północnego [La végétation de la grande Forêt de Kurpie à base des rapports gèobotaniques du Secteur Septentrional], Acta Soc. Bot. Pol., 4. (34): 719-752.

Faliński J.B. \& Bartel J., 1965, Quelques groupements végétaux dans le Basin de la Riviére Ełk. Mater. Zakł. Fitosoc. Stos. UW, vol. 6: 97-108.

Fijałkowski D., 1993, Lasy Lubelszczyzny [Forests of the Lublin Region]. Lubelskie Tow. Naukowe, Lublin.

Fijałkowski D. \& Górski J., 1968, Stosunki ekologiczne i fitosocjologiczne siedlisk mącznicy lekarskiej (Arctostaphylos uva-ursi L.) pod Zaklikowem w województwie lubelskim [Die ökologischen und phytosociologischen Verhältnisse von Arctostaphylos uva-ursi L. Gesellschaften bei Zaklików in der Wojewodschaft Lublin], Fragm. Flor. Geobot. 14(4): 433-441.

Gawłowska J., 1964, Mącznica lekarska - Arctostaphylos uva-ursi L. w Polsce, jej zasoby i ochrona [The bearberry, Arctostaphylos uva-ursi L., in Poland, its resources and protection], Ochrona Przyrody 30: 23-50.
Gugnacka-Fiedor W. \& Adamska E., 2010, The preservation state of the flora and vegetation of the artillery range near the city of Toruń, Ecological Questions 12 (this issue).

Kępczyński K., 1965, Szata roślinna Wysoczyzny Dobrzyńskiej [Die Pflanzenwelt des Diluvialplateau's von Dobrzyń], Wydawnictwa UMK, Toruń.

Kobendza R., 1930, Stosunki fitosocjologiczne Puszczy Kampinoskiej [Les rapports phytosociologiques dans l'ancienne grande Forêt de Kampinos], Planta Polonica vol. II, Materiały do flory polskiej [Contributions à la flore de la Pologne], Tow. Naukowe Warszawskie, Warszawa.

Kovach W., 1985-1999, MVSP PLUS version 3.1, Pentraeth, UK.

Kujawa-Pawlaczyk J., 2004, Suche wrzosowiska (CallunoGenistion, Pohlio-Callunion, Calluno-Arctostaphylion) [Dry heathlands (Calluno-Genistion, Pohlio-Callunion, Calluno-Arctostaphylion)], [in:] J. Herbich (ed.) Murawy, łąki, ziołorośla, wrzosowiska, zarośla, Poradniki ochrony siedlisk i gatunków Natura 2000 - podręcznik metodyczny, tom 3 [Grasslands, meadows, tall herb vegetation, heaths, thickets, Methodical guides to conservation of habitats and species, Natura 2000, Volume 3], Ministerstwo Środowiska, Warszawa: 32-48.

Matuszkiewicz J. M., 1993. Zespoły leśne Polski [Forest Plant Associations of Poland], Wydawnictwo Naukowe PWN, Warszawa.

Matuszkiewicz W., 2007, Przewodnik do oznaczania zbiorowisk roślinnych Polski [A guide for identification of plant communities in Poland], PWN, Warszawa.

Meller I., 1994, Szata roślinna projektowanego rezerwatu pod nazwą Kły [Vegetation cover of the designed reserve called Kły], Master Thesis, Faculty of Biology and Earth Sciences, Dept. of Taxonomy and Plant Geography, NCU, Torun.

Piernik A., 2008, Metody numeryczne w ekologii na przykładzie zastosowań pakietu MVSP do analiz roślinności [Numerical methods in ecology on examples of the use of package MVSP for analyses of vegetation], Wydawnictwo Naukowe UMK, Torun.

Spałek K., 1996, Dwa nowe stanowiska Arctostaphylos uva-ursi L. na Śląsku Opolskim [New location of Arctostaphylos uva-ursi L. in Opole Silesia], Opolskie Tow. Przyj. Nauk, Zesz. Przyr. 31: 5-10.

Tüxen R., 1942, Calluna-Arctostaphylos uva ursi-Ass., Rdbf. D. Zentralstelle f. Vegetationskartierung, Stolzenau: 64-66.

Werdyn L., 1964, Materiały do rozmieszczenia Arctostaphylos uva-ursi L. na Niżu Polskim [Notes on distribution of Arctostaphylos uva-ursi L. in the Polish lowland], Bad.Fizjogr. nad Polską Zach. 14: 127-143. 